\title{
The Association of Insulin Resistance and Metabolic Syndrome with Age- Related Hearing Loss
}

Ning-Chia Chang ${ }^{1-3}$, Chen-Yu Chien ${ }^{1,3}$, Meng-Hsuan Hsieh ${ }^{4,5}$, Wen-Yi Lin ${ }^{2}$ and Kuen-Yao Ho ${ }^{1,6 *}$

${ }^{1}$ Department of Otorhinolaryngology, School of Medicine, College of Medicine, Kaohsiung Medical University, Kaohsiung, Taiwan

${ }^{2}$ Department of Occupational Medicine, Kaohsiung Municipal Hsiao-Kang Hospital, Kaohsiung, Taiwan

${ }^{3}$ Department of Otorhinolaryngology, Kaohsiung Municipal Hsiao-Kang Hospital, Kaohsiung, Taiwan

${ }^{4}$ Department of Internal Medicine, School of Medicine, College of Medicine, Kaohsiung Medical University, Kaohsiung, Taiwan

${ }^{5}$ Department of Preventive Medicine, Kaohsiung Medical University Hospital, Kaohsiung, Taiwan

${ }^{6}$ Department of Otorhinolaryngology, Kaohsiung Medical University Hospital, Kaohsiung, Taiwan

\begin{abstract}
Objectives: Metabolic syndrome (MetS) is a complex metabolic disease characterized by central obesity, impaired glucose metabolism, dyslipidemia, arterial hypertension, insulin resistance, and high-sensitivity C-reactive protein. Some factors associated with MetS may increase the risk of hearing loss; we hypothesized that cases with MetS would have an acceleration of hearing loss compared to those without MetS. The purpose of this study was to analyze the association of insulin resistance and MetS with hearing impairments and to prove our hypothesis.
\end{abstract}

Methods: This study was a retrospective study performed in a medical center. The subjects in this study were the employees from a single company. Homeostasis Model Assessment for Insulin Resistance (HOMA-IR) was used as the index of insulin resistance. The pure tone audiometric data of a 10-year-period were retrieved from the computerized database. The 10-year shifts of average hearing levels, including conventional frequencies $(0.5,1$, $2 \mathrm{kHz})$ and high frequencies $(4,6,8 \mathrm{kHz})$, were used to analyze the correlations with HOMA-IR and MetS. Linear regressions were used to estimate the coefficients of age, sex, HOMA-IR, and metabolic syndrome to hearing level shifts. The level of statistical significance was set as $p<0.05$.

Results: Data from169 cases, including 162 men and 7 women, were analyzed in this study. Age was the most relevant factor to hearing loss. After adjusting for age and sex, HOMA-IR was found to be associated with a high-frequency hearing level shift (coefficients $=0.156, p=0.035$ ), while HOMA-IR was not found to be significantly associated with conventional frequency hearing loss. However, MetS was not found to be significantly associated with either conventional or high frequency hearing level shifts.

Conclusions: Insulin resistance is associated with the acceleration of age-related hearing loss, while MetS is not shown to increase the risk of hearing loss. Based on the findings of this study, agents that help lower insulin resistance may play a role in the deceleration of age-related hearing impairment.

Keywords: Insulin resistance; HOMA-IR; Diabetes; Metabolic syndrome; Pure tone audiometry; Hearing loss; Presbycusis

\section{Introduction}

Metabolic Syndrome (MetS) is a complex metabolic disease characterized by central obesity, impaired glucose metabolism, dyslipidemia, arterial hypertension, insulin resistance, and highsensitivity C-reactive protein [1-3]. MetS increases the risk of cardiovascular disease and predicts a high probability of the development of diabetes (if not already present) $[2,3]$.

Some components of MetS are reported to be associated with hearing loss. Hypertriglyceridemia was found to increase the risk of noise-induced hearing loss in our previous study [4]. It was postulated that the free radicals and Reactive Oxygen Species (ROS) generated during the metabolism of excess triglyceride would increase the necrosis/apoptosis of inner ear cells and lead to hearing loss. The root cause of the free radicals and ROS is an impaired glucose metabolism, which may disturb the normal metabolic pathway and decease primary energy generation from glucose. Instead, lipid peroxidation and protein oxidation may be alternative pathways to provide sufficient energy for cells to use. However, the process of protein oxidation and lipid peroxidation releases many unfavorable by-products, free radicals and ROS, leading to damage of the auditory physiology [5-7].

Diabetes mellitus is the ultimate outcome of glucose metabolic dysfunction. Prior studies reported that patients with type 2 diabetes had an increased risks of hearing loss [5,6,8-16]. Microangiopathy, advanced glycation end products, and ROS are the major mechanisms that are believed to explain diabetes-associated hearing loss [6]. It was reported that the prevalence of hearing loss increased with the duration of diabetes [10]. Because insulin resistance is the initiation and core pathogenesis of type 2 diabetes, we investigated the association of insulin resistance with hearing impairment.

There is little literature discussing the relationship between MetS and hearing loss. Because some factors associated with MetS may increase the risk of hearing loss, we hypothesized that cases with MetS would have an acceleration of hearing loss compared to those without MetS. The purpose of this study was to answer the question regarding the association of insulin resistance with hearing impairments and to prove our hypothesis.

*Corresponding author: Kuen-Yao Ho, MD, Department of Otorhinolaryngology, Kaohsiung Medical University Hospital, No.100, Tzyou 1st Road, Kaohsiung 807 , Taiwan, Tel: +886-7-3208264; Fax: +886-7-3208264; E-mail: kuyaho@kmu.edu.tw

Received: June 09, 2014; Accepted: September 24, 2014; Published: October 02, 2014

Citation: Chang NC, Chien CY, Hsieh MH, Lin WY, Ho KY (2014) The Association of Insulin Resistance and Metabolic Syndrome with Age-Related Hearing Loss. Diabetes Metab 5: 440 doi:10.4172/2155-6156.1000440

Copyright: (c) 2014 Chang NC, et al. This is an open-access article distributed under the terms of the Creative Commons Attribution License, which permits unrestricted use, distribution, and reproduction in any medium, provided the original author and source are credited. 


\section{Materials and Methods}

\section{Ethical considerations}

The materials were from the database of the Department of Preventive Medicine in our hospital. No private personal information was identifiable in the data. This study was approved by the institutional review board of our hospital (IRB approval No.: KMHKIRB-A96062580).

\section{Subjects}

The subjects of this study were the clients who received annual health examinations performed by the Department of Preventive Medicine, Kaohsiung Medical University Hospital during the years from 2002 to 2012. The subjects in this study were the employees from a single company. The health examinations included routine blood work, regular blood chemistries, blood pressures, general physical examination, otoscopic examination and pure tone audiometry (PTA). During the health examinations, the subject's High-Density Cholesterol (HDL) level and waist circumference were measured. The fasting insulin level was measured as part of another study on MetS in 2008. During the health examination, the subjects were asked about their personal medical history, habitual use of drugs, and specific noise exposure experience. All the subjects claimed to have had no occupational noise exposure over $80 \mathrm{dBA}$. Subjects with ear diseases that may affect hearing thresholds (e.g., otitis media, cholesteatoma, ear canal stenosis, etc.) and those who used potentially ototoxic drugs (e.g., aspirin, quinolones, aminoglycosides, etc.) were excluded from this study.

\section{Audiological assessment}

Pure-tone audiometry was performed using a Beltone 120 audiometer (Beltone Electronics Corp. Chicago, IL, USA) and TDH 50-P earphones (Beltone Electronics Corp. Chicago, IL, USA) calibrated to ISO 389 (1975). All of the audiometric tests were performed with standard procedures by trained technicians in sound-attenuating booths that met the requirements of the Council of Labor Affairs, Executive Yuan, Taiwan. The audiometric data were recorded at the frequencies 500, 1000, 2000, 3000, 4000, 6000, and $8000 \mathrm{~Hz}$. Hearing levels were computed based on these frequencies. The conventional hearing level was defined as the average hearing threshold recorded at 500,1000, and $2000 \mathrm{~Hz}$ (HL512). The high-frequency hearing level was defined as the mean hearing threshold recorded at 4000, 6000 and $8000 \mathrm{~Hz}$ (HL468). The hearing levels recorded during 2002 were used as baseline data. The differences of hearing levels between 2012 and 2002 denoted the deteriorations of hearing over a 10 -year period.

\section{Definition of metabolic syndrome}

Based on the modified definition of metabolic syndrome by the Health Promotion Administration, Ministry of Health and Welfare, Taiwan (ROC), a case with MetS was defined when the subject met at least three of the following five criteria: (1) blood pressure of at least $130 / 85 \mathrm{mmHg}$ or receiving treatment for hypertension; (2) serum triglyceride (TG) of at least $150 \mathrm{mg} / \mathrm{dL}$; (3) high-density lipoproteins (HDL) cholesterol $<40 \mathrm{mg} / \mathrm{dL}$ in men and $<50 \mathrm{mg} / \mathrm{dL}$ in women; (4) fasting glucose $\geq 100 \mathrm{mg} / \mathrm{dL}$ or receiving treatment for diabetes; and (5) waist circumference $\geq 90 \mathrm{~cm}$ in men and $\geq 80 \mathrm{~cm}$ in women. With the measurement of insulin level, the case of MetS was defined based on the data recorded in 2008.

\section{Index of insulin resistance}

The homeostasis model assessment for insulin resistance (HOMA-
IR) was used as the index of insulin resistance in this study. HOMAIR is obtained as fasting serum insulin level $(\mu \mathrm{U} / \mathrm{mL}) \times$ fasting plasma glucose level $(\mathrm{mg} / \mathrm{dL}) / 405$. This index was used as a parameter in the analysis of risk factors for hearing loss.

\section{Statistical Analysis}

All the data were computerized and analyzed with the SPSS statistics software package version 16.0 (SPSS Inc., Chicago, IL, USA). Continuous data were analyzed by independent sample Student's t-tests. Categorical data were computed by two-sided $\chi^{2}$ tests or Fisher's exact tests. The associations of age, sex, fasting glucose level, fasting triglyceride level, cholesterol levels, waist circumference, blood pressures, fasting insulin level, and hearing level shifts were analyzed. Linear regressions were used to estimate the coefficients of age, sex, HOMA-IR, and metabolic syndrome to hearing level shifts. The level of statistical significance was set as $\mathrm{p}<0.05$.

\section{Results}

In total, 169 cases with comprehensive data from 2002 to 2012, including 7 women and 162 men, were enrolled in this study. The average age was $49.22 \pm 9.34$ years in 2012. The gender-based descriptive data of MetS (fasting triglyceride level, fasting plasma glucose level, HDL level, waist circumference, and blood pressures), fasting insulin level, HOMA-IR, and hearing levels (HL512 and HL468 in the years 2002 and 2012) are summarized in Table 1 . Men had significantly larger

\begin{tabular}{|c|c|c|c|c|}
\hline & Total & Female & Male & P value $^{\mathbf{a}}$ \\
\hline Number & 169 & 7 & 162 & \\
\hline Age (year) & $49.22 \pm 9.34$ & $50.86 \pm 6.44$ & $47.15 \pm 9.45$ & 0.523 \\
\hline $\begin{array}{l}\text { Fasting Triglyceride } \\
(\mathrm{mg} / \mathrm{dL})\end{array}$ & $145.42 \pm 118.77$ & $87.29 \pm 56.95$ & $147.93 \pm 120.19$ & 0.187 \\
\hline $\begin{array}{l}\text { Total Cholesterol } \\
(\mathrm{mg} / \mathrm{dL})\end{array}$ & $202.96 \pm 34.66$ & $220.43 \pm 36.51$ & $202.20 \pm 34.50$ & 0.174 \\
\hline HDL (mg/dL) & $51.02 \pm 11.62$ & $70.50 \pm 8.68$ & $50.18 \pm 11.00$ & $<0.001^{* *}$ \\
\hline $\begin{array}{l}\text { Waist Circumference } \\
(\mathrm{cm})\end{array}$ & $83.83 \pm 8.30$ & $68.71 \pm 7.89$ & $84.49 \pm 7.69$ & $<0.001^{* *}$ \\
\hline \multicolumn{5}{|l|}{$\begin{array}{l}\text { Blood Pressure } \\
(\mathrm{mmHg})\end{array}$} \\
\hline Systolic & $124.04 \pm 14.34$ & $110.71 \pm 16.91$ & $124.61 \pm 14.00$ & $0.012^{*}$ \\
\hline Diastolic & $77.78 \pm 11.74$ & $68.86 \pm 9.92$ & $78.17 \pm 11.69$ & $0.040^{*}$ \\
\hline Metabolic syndrome & & & & 0.68 \\
\hline yes & 43 & 1 & 42 & \\
\hline no & 126 & 6 & 120 & \\
\hline $\begin{array}{l}\text { Fasting plasma } \\
\text { glucose (mg/dL) }\end{array}$ & $104.06 \pm 20.89$ & $95.57 \pm 6.63$ & $104.43 \pm 21.22$ & 0.273 \\
\hline $\begin{array}{l}\text { Fasting Insulin Level } \\
(\mu \mathrm{U} / \mathrm{mL})\end{array}$ & $8.73 \pm 6.67$ & $6.34 \pm 2.42$ & $8.83 \pm 6.77$ & 0.336 \\
\hline HOMA-IR & $2.27 \pm 1.82$ & $1.51 \pm 0.67$ & $2.31 \pm 1.85$ & 0.26 \\
\hline \multicolumn{5}{|l|}{ HL512 (dB) } \\
\hline Year 2002 & $15.22 \pm 5.32$ & $13.81 \pm 1.59$ & $15.28 \pm 5.42$ & 0.477 \\
\hline Year 2012 & $15.36 \pm 7.74$ & $12.14 \pm 3.60$ & $15.50 \pm 7.85$ & 0.262 \\
\hline shift & $0.15 \pm 5.34$ & $-1.67 \pm 3.26$ & $0.23 \pm 5.61$ & 0.378 \\
\hline \multicolumn{5}{|l|}{ HL468 (dB) } \\
\hline Year 2002 & $21.58 \pm 15.04$ & $13.10 \pm 3.36$ & $21.95 \pm 15.24$ & 0.128 \\
\hline Year 2012 & $28.90 \pm 17.79$ & $14.88 \pm 6.93$ & $29.50 \pm 17.88$ & $0.01^{*}$ \\
\hline shift & $7.31 \pm 7.08$ & $1.79 \pm 6.55$ & $7.55 \pm 7.02$ & $0.034^{*}$ \\
\hline
\end{tabular}

a: the $p$ values were obtained by $t$ tests, except the metabolic syndrome, which was obtained by Fisher exact test.

*: p level $<0.05$

$* *$ : p level $<0.01$

Table 1: Descriptive Characteristics of the population in this study. 


\begin{tabular}{|c|c|c|c|c|c|c|c|c|}
\hline & \multicolumn{4}{|c|}{ HL512a } & \multicolumn{4}{|c|}{ HL468 ${ }^{b}$} \\
\hline & correlation & $\mathrm{p}$ & coefficients & $\mathrm{p}$ & correlation & $\mathrm{p}$ & coefficients & $\mathrm{p}$ \\
\hline Age & 0.268 & $<0.001^{* *}$ & \multirow{2}{*}{\multicolumn{2}{|c|}{ NA }} & 0.255 & $0.001^{* \star}$ & \multirow{2}{*}{\multicolumn{2}{|c|}{ NA }} \\
\hline Sex & 0.068 & 0.378 & & & 0.163 & $0.034^{*}$ & & \\
\hline HOMA-IR & 0.038 & 0.628 & 0.028 & 0.713 & 0.173 & $0.025^{\star}$ & 0.156 & $0.035^{*}$ \\
\hline Metabolic syndrome (N/Y) & 0.023 & 0.764 & 0.016 & 0.826 & 0.054 & 0.485 & 0.048 & 0.527 \\
\hline $\begin{array}{l}\text { Elevated Fasting Plasma } \\
\text { glucose }\end{array}$ & 0.199 & $0.009^{*}$ & 0.145 & 0.580 & 0.125 & 0.105 & 0.060 & 0.433 \\
\hline $\begin{array}{l}\text { Elevated Fasting } \\
\text { Triglyceride level }\end{array}$ & -0.095 & 0.220 & -0.076 & 0.314 & -0.033 & 0.670 & -0.020 & 0.793 \\
\hline Low HDL level & -0.039 & 0.618 & -0.049 & 0.511 & -0.004 & 0.957 & -0.014 & 0.850 \\
\hline Central Obesity & 0.009 & 0.911 & 0.014 & 0.855 & 0.079 & 0.306 & 0.084 & 0.262 \\
\hline Elevated Blood Pressure & 0.018 & 0.814 & -0.040 & 0.602 & 0.067 & 0.389 & -0.005 & 0.950 \\
\hline
\end{tabular}

a: average hearing level at frequency 500,1000 and $2000 \mathrm{~Hz}$ of bilateral ears

b: average hearing level at frequency 4000,6000 and $8000 \mathrm{~Hz}$ of bilateral ears

Regression coefficients: parameters controlled for age; for non-sex dependent parameters (i.e. HOMA-IR, glucose level, triglyceride level, and blood pressure), sex were also controlled.

For the components of metabolic syndrome, binary observations were used: $N=0$ and $Y=1$.

*: $\mathrm{p}$ level<0.05

**: p level<0.01

Table 2: Correlations of HOMA-IR and Metabolic Syndrome with 10-year-shift of hearing levels.

waist circumferences and lower HDL levels, which were inherently different between the sexes. Hence, these two parameters were adjusted by sex when defining MetS. Blood pressures were higher in men than in women by approximately $10 \mathrm{mmHg}$, regardless of systolic or diastolic blood pressure. No significant difference in the distribution of MetS between sexes was noted. The sex-related difference was a potential factor that may affect the shift of hearing level in the 10-year period, especially for high frequency hearing. Men had an average 7.55 \pm 7.02 $\mathrm{dB}$ shift at HL468 over 10 years, much higher in comparison with the average HL468 shift of $1.79 \pm 6.55 \mathrm{~dB}$ for women (Table 1). However, there was no significant sex-related difference in the shift of HL512.

In the analysis of the factors associated with hearing loss, age was found to be the most important factor relating to hearing level shifts. Age showed significant associations $(\mathrm{p} \leq 0.001)$, with 10 -year hearing level shifts with correlation constants of 0.268 for HL512 and 0.255 for HL468 (Table 2). The elevated plasma fasting glucose level seemed to have some effect in the 10-year shift of HL512. The analyses of the other MetS parameters failed to find any significant association with hearing level shifts. Insulin resistance (HOMA-IR) was found to have some influence in the shift of HL468 (correlation constant $=0.173, \mathrm{p}=0.025$ ). Linear regressions were used to analyze the degree of influence of each factor to the shift of hearing levels. After controlling for the sex of nonsex dependent parameters (i.e., HOMA-IR, glucose level, triglyceride level, and blood pressure) and age, the significance of the association between glucose levels and hearing level shifts disappeared. However, HOMA-IR remained and demonstrated an association with the shift of HL468 (coefficients $=0.156, p=0.035$ ), despite the controlling for age and sex.

\section{Discussion}

In this study, we found that HL512 was relatively stable over a period of 10 years. The shift of HL512 was minimal $(0.15 \pm 5.34 \mathrm{~dB})$ over 10 years and did not greatly affect one's daily life or have a specific significance in epidemiology. In contrast, the shift of HL468 over 10 years was greater $(7.31 \pm 7.05 \mathrm{~dB})$, indicating that high-tone hearing loss occurs with aging, which could be considered as presbycusis. Age was found to be the most important determinant of a hearing level shift, especially for HL468. Thus, high-tone hearing loss with aging (presbycusis) is accelerated in aged people but not at a consistent rate.
HOMA-IR was found to have a positive association with the shift of HL468, and this finding could be interpreted as insulin resistance relating to an acceleration of aging hearing impairment. The mechanism of the influence of insulin resistance to age-related hearing impairment is still unclear. There are few reports discussing the interactions between MetS and hearing loss and even fewer reports focus on the associations of insulin resistance with hearing loss. However, many studies investigating the association of diabetes mellitus with hearing loss have been published [5,6,8-16]. Individuals with insulin resistance are generally considered to be candidates for type 2 diabetic patient statuses or at pre-diabetic status. The mechanism of insulin resistance related acceleration of presbycusis should be similar or even identical to that of diabetes-associated hearing loss. In the condition of insulin resistance, the metabolism of glucose is disturbed, decreasing energy generation from glycolysis. However, the auditory system requires high energy utilization for its complex signal processing. The energy is thus acquired from alternative origins: protein oxidation and lipid peroxidation [5-8]. During the processing of protein oxidation and lipid peroxidation, a large amount of free radicals and Reactive Oxygen Species (ROS) are generated. The free radicals and ROS then cause mitochondria dysfunction and mitochondrial DNA (mtDNA) impairments and lead to cellular degeneration $[8,17]$. In the inner ear, the result of this process causes hearing impairment. Another aspect of insulin resistance is impaired insulin signaling in the tissue. Impaired insulin metabolic signaling results in impaired glucose uptake, endothelial dysfunction, impaired angiogenesis, metabolic inflexibility, vascular stiffness, and atherosclerosis [18]. The blood flow is disrupted, causing ischemic events of the cochlea that lead to hair cell apoptosis or necrosis, ultimately resulting in hearing loss.

Metabolic syndrome, in contrast to our hypothesis, was not found to be associated with age-related hearing loss. The explanation may be that some components of MetS under the definition that we used were not proven risk factors for hearing loss. Our previous study revealed that hypertriglyceridemia may increase the risk of noise-induced hearing loss by $28 \%$ [4], and the mechanism may be similar for age-related hearing loss. Diabetes mellitus and/or hyperglycemia were found to be related to the acceleration of hearing loss in several studies, and insulin resistance were found to be related to the acceleration of hearing loss in the present study $[6,8,10,11]$. The impact of hypercholesterolemia 
Citation: Chang NC, Chien CY, Hsieh MH, Lin WY, Ho KY (2014) The Association of Insulin Resistance and Metabolic Syndrome with Age-Related Hearing Loss. J Diabetes Metab 5: 440 doi:10.4172/2155-6156.1000440

on hearing loss is controversial. In an early study, hypercholesterolemia was not found to be associated with hearing loss [19]. However, the recent study revealed that low-density lipoprotein may be important in auditory dysfunction [20]. There was no evidence supporting hypertension and central obesity increasing the risk of hearing loss. There were some participants with hypertension, central obesity and low HDL levels diagnosed with MetS. While these cases did not carry hypertriglyceridemia or hyperglycemia, which are considered to be risk factors for hearing loss, it was reasonable to conclude that MetS was not associated with age-related hearing loss.

One limitation of this study was that we lacked an established database for the normalized level of age-specific hearing impairment in our country. Without such data, we could not determine a cutoff level to identify whether a case has excessive hearing loss by his/ her age. Not having these data prevented us from obtaining the odds ratios of influence of HOMA-IR to age-related hearing loss by logistic regressions. The establishment of such a database may help in future analyses.

\section{Conclusions}

The current study found that insulin resistance (HOMA-IR) was associated with the acceleration of age-related hearing loss. Although insulin resistance is a part of MetS in some definitions, it was not adopted in our national definition of MetS. Metabolic syndrome was not found to be significantly associated with age-related hearing loss in this study. A cohort study on the change of hearing status may provide a clearer profile for the influences of insulin resistance status and the development of diabetes and MetS on presbycusis. Based on the findings of this study, agents that help lower insulin resistance may play a role in the deceleration of age-related hearing impairment.

\section{References}

1. Hristova MG (2013) Metabolic syndrome--from the neurotrophic hypothesis to a theory. Med Hypotheses 81: 627-634.

2. Alberti KG, Zimmet P, Shaw J (2006) Metabolic syndrome--a new world-wide definition. A Consensus Statement from the International Diabetes Federation. Diabet Med 23: 469-480.

3. Eckel RH, Grundy SM, Zimmet PZ (2005) The metabolic syndrome. Lancet 365: $1415-1428$.

4. Chang NC, Yu ML, Ho KY, Ho CK (2007) Hyperlipidemia in noise-induced hearing loss. Otolaryngol Head Neck Surg 137: 603-606.
5. Aladag I, Eyibilen A, Guven M, Atis O, Erkorkmaz U (2009) Role of oxidative stress in hearing impairment in patients with type two diabetes mellitus. J Laryngol Otol 123: 957-963.

6. Hong O, Buss J, Thomas E (2013) Type 2 diabetes and hearing loss. Dis Mon 59: $139-146$

7. Martin SD, McGee SL (2014) The role of mitochondria in the aetiology of insulin resistance and type 2 diabetes. Biochim Biophys Acta 1840: 1303-1312.

8. Frisina ST, Mapes F, Kim Set, Frisina D R, Frisina RD (2006) Characterization of hearing loss in aged type II diabetics. Hearing research 211:103-113.

9. Jang TW, Kim BG, Kwon YJ, Im HJ (2011) The association between impaired fasting glucose and noise-induced hearing loss. J Occup Health 53: 274-279.

10. Sunkum AJ, Pingile S (2013) A clinical study of audiological profile in diabetes mellitus patients. Eur Arch Otorhinolaryngol 270: 875-879.

11. Horikawa C, Kodama S, Tanaka S, Fujihara K, Hirasawa R, et al. (2013) Diabetes and risk of hearing impairment in adults: a meta-analysis. $\mathrm{J}$ Clin Endocrinol Metab 98: 51-58.

12. Mozaffari M, Tajik A, Ariaei N, Ali-Ehyaii F, Behnam H (2010) Diabetes mellitus and sensorineural hearing loss among non-elderly people. East Mediterr Health J 16: 947-952.

13. Uchida Y, Sugiura S, Ando F, Nakashima T, Shimokata H (2010) Diabetes reduces auditory sensitivity in middle-aged listeners more than in elderly listeners: a population- based study of age-related hearing loss. Medical science monitor16:PH63-8.

14. Akinpelu OV, Mujica-Mota M, Daniel SJ (2014) Is type 2 diabetes mellitus associated with alterations in hearing? A systematic review and meta-analysis. Laryngoscope 124: 767-776.

15. Bainbridge KE, Hoffman HJ, Cowie CC (2011) Risk factors for hearing impairment among U.S. adults with diabetes: National Health and Nutrition Examination Survey 1999-2004. Diabetes Care 34: 1540-1545.

16. Lerman-Garber I, Cuevas-Ramos D, Valdés S, Enríquez L, Lobato M, et al (2012) Sensorineural hearing loss--a common finding in early-onset type 2 diabetes mellitus. Endocr Pract 18: 549-557.

17. Le T, Keithley EM (2007) Effects of antioxidants on the aging inner ear. Hear Res 226: 194-202.

18. Aroor AR, McKarns S, Demarco VG, Jia G, Sowers JR (2013) Maladaptive immune and inflammatory pathways lead to cardiovascular insulin resistance. Metabolism 62: 1543-1552.

19. Saito T, Sato K, Saito H (1986) An experimental study of auditory dysfunction associated with hyperlipoproteinemia. Arch Otorhinolaryngol 243: 242-245.

20. Thakur JS, Mohindroo NK, Vasanthalakshmi MS, Kashyap N, Azad RK, et al. (2012) Auditory brainstem evoked responses in hyperlipidaemia: effect of various lipid fractions on auditory function. The Journal of laryngology and otology 126: 249-256. 